\title{
Clothing Communication via Social Media: A Decision Tree Predictive Model
}

\author{
So Young Song, University of Tennessee, USA \\ Erin Cho, Parsons, The New School for Design, USA \\ Youn-Kyung Kim, University of Tennessee, USA \\ Theresa Hyunjin Kwon, Seoul National University, South Korea
}

Keywords: Clothing Communication, Social Media, Decision Tree, Personality

\section{Research Background}

Consumers are increasingly using social media (SM) as an important source of information and as a way to communicate about clothing. SM platforms such as Twitter, Facebook, and YouTube have already been evaluated as successful business take-off tools by numerous clothing brands such as Burberry, Nordstrom, and ASOS (Kim \& Ko, 2012). While personality differences have been examined in relation to SM use, one area that remains unexplored is the influence of personality traits on information exchange and dialogue about clothing (Hart et al., 2015). The current study addresses this issue by linking personality traits to clothing communication via social media (CCSM) measured by SM usage for the clothing product category. Evidence suggests that SM users are goal-directed in their usage, and seek gratification to satisfy their individual needs that are derived from their personality traits (Orchard et al., 2014), which is reflected by the "uses and gratification" (U\&G) theory (Katz et al., 1974). Drawing upon this theory, we propose to build a decision tree predictive model of CCSM based on personality traits (i.e., friendship, exhibitionism, self-love, femininity, openness, masculinity, intelligence, superiority, and exploitativeness). We also investigate whether demographic characteristics (i.e., age, gender, income, employment status, and residential area) can forecast CCSM. This decision tree model will identify important personality traits in predicting different CCSM segments.

\section{Methods and Results}

A total of 370 usable responses were collected from online consumer panelists. Nine pertinent personality traits were identified from the literature and used as predictors of CCSM segments such as friendship, exhibitionism, openness, femininity, masculinity, intelligence, selflove, superiority, and exploitativeness. The scale items for CCSM were developed by the researchers with two main questions: the frequency of discussing and the extent to which they are influenced by the discussion for the clothing products. The reliabilities of all scale items measured by Cronbach's alpha coefficients exceeded 0.8. The results from an univariate test and a two-sample t-test between low and high CCSM groups indicated that there was a significant difference based on SM usage: $F(1,368)=1854.84, p<.001 ; t(368)=43.07, p<.001$; high group $\mathrm{M}=5.66, \mathrm{SD}=1.15$; low group $\mathrm{M}=1.50, \mathrm{SD}=.69$; and mean difference $=4.16$. Using a recursive partitioning method, we applied a version of Classification and Regression Trees (CART), available in R statistical software for creating a binary decision tree. The resultant classification tree of CCSM was comprised of eight terminal nodes: four high CCSM segments

Page 1 of 2 
and four low CCSM segments. The decision tree started with a root node $(\mathrm{n}=370)$ that split into two branches by friendship ( $\geq 6.2, \mathrm{n}=89,24 \% ;<6.2, \mathrm{n}=281,76 \%$ ), successively leading to further nodes by exhibitionism $(\geq 4.4)$, femininity $(\geq 5.4)$, self-love $(\geq 5.8)$, and exploitativeness $(<5.2)$ and produced an optimal predictive model forecasting CCSM segments.

The result indicated that high levels of consumers' personality traits such as friendship, exhibitionism, self-love, and femininity predicted high CCSM segments. The first root node was split by friendship, suggesting that friendship is the most important factor in predicting CCSM ( $\geq 6.2, \mathrm{n}=89,24 \%)$. The largest segment $(\mathrm{n}=137,37 \%)$, categorized as a low CCSM group, was explained by low levels of friendship $(<6.2)$, exhibitionism $(<3.6)$, and femininity $(<5.4)$. However, demographic variables (i.e., age, gender, income, employment status, and residential area) were found to be insignificant predictors in our decision tree model. We validated the final model's performance by conducting error matrix and receiver-operating characteristic (ROC) analyses. The results showed overall error rate of $24 \%$ and AUROCC 0.80 with satisfactory accuracy and low misclassification rate. Finally, using personality trait inputs, Gini variable importance by random forest algorithms, computed by building five hundreds of decision trees $($ ntree $=500)$, confirmed the relative importance of variables corresponding to our tree model.

\section{Implication and Conclusions}

Our decision tree model provides information not only on efficiently segmenting populations into CCSM subsets, but also on efficiently targeting and maximizing clothing retailers' distribution of SM marketing resources. The results suggest that such personality traits of friendship, exhibitionism, and self-love are best predictors of high CCSM segments. We also found that the effect of gender on CCSM was not significant but the personality trait of femininity was important in predicting CCSM segments. That is, a high CCSM segment group is likely to have more face-to-face and online friends, like to flaunt themselves, love to look at themselves, and tend to be more feminine.

Clothing retailers can enhance SM clothing communication by incorporating personality traits of friendship, exhibitionism, and self-love. Specifically, we suggest retailers should focus on short-form video content in boosting consumers' engagement related to apparel products, brands, or functional message that they wants to deliver. Providing self-promotion opportunities for consumers to post short-form social video (e.g., Vine's six-second-loop video format), or audio-visual storytelling will enhance their friendship (i.e., make more online friends), meet their needs to show off, and generate self-satisfaction. Further, clothing retailers should be aware that targeting specific segments based on demographic variables exclusively is not adequate. Based on our results, personality traits are more important factors in predicting CCSM than is demographic information. In sum, our study contributes to social and psychological aspects of social media usage and provides a new analytical approach predicting clothing communication via social media.

\section{References}

Seidman, G. (2013). Self-presentation and belonging on Facebook: How personality influences social media use and motivations. Personality and Individual Differences, 54(3), 402-407. 